2) There are misgivings of complications when severe clouding of consciousness are lasting for a long time in the case of severe head trauma which have no operation adaptation. The authors experienced that tracheotomy, change of position and therapy of lung complication. In this communication, some blood properties of the case of clouding of consciousness for a long time are reported.

\title{
61. Hemodynamics of the Brain Stem under the Head Injury
}

Experimental Studies by means of the Method of Determination of the Hemodynamics of the Galen's Vein

\author{
Rokuro Takayama, Kihachiro Masuda, Sakae Fukuda, Hideyuki Hirai, \\ Isao Katayama, Shigeo Toya, Shiro ShimizU, Motohiro TanaKa \\ Department of Surgery, School of Medicine, Keio University \\ and Nobuaki SHibuYa \\ Department of Surgery, Saiseikai Chuoh Hospital
}

Since the first report of our experimental research on the hemodynamics of the Galen's vein at the 17th Congress of the Japan Neurosurgical Society, we have pursued serial experiments on the same subject. On this occasion we are to report on the results of experiments with regard to closed head injury.

Small trephine openings are made on the vertex and occipital region of the dogs instead of the temporal craniotomy regularly followed previously, and polyethylene tubes are inserted into the superior sagittal sinus and the Galen's vein for comparative studies.

Vicissitudes of respiration and blood pressure are recorded simultaneously.

1) After the injury the blood flow of the superior sagittal sinus and the Galen's vein shows a transient increase and then declines gradually. The change is more prominent in the Galen's vein.

2) Dogs subjected to combination of experimental hemorrhage and head injury shows blood flow increase in the Galen's vein and the superior sagittal sinus in the early stage, and then the blood flow decrease gradually especially in the Galen's vein.

3) In dogs subjected to experimental asphyxia, the blood flow of the Galen's vein and the superior sagittal sinus shows increase initially accompanying the rise of blood pressure. In the next phase the blood flow declines rapidly, and the animal gradually deceases. These changes are more marked in the Galen's vein than in the superior sagittal sinus. 


\title{
SUMMARY
}

Considerable amount of works has been reported on the study of cerebral circulation, but not so many reports are available on the subject of stem circulation.

The authers performed experimental studies on the hemodynamics of the Galen's vein of dogs with acute closed head injury, and the results are reported in this paper.

\section{Serial Observations of Eye Grounds in Patients of Head Injury}

\author{
Shigeo Watanabe and *Yoshiro Takeuchi \\ Chukyo General Hospital
}

We have attempted to investigate a change of artery and vein of eye ground, in order to determine a clinical relation between patient's condition and findings of retinal vessels, using Portable Fundus Camera for head injury.

1) Contraction of artery and dilatation of vein in retina were observed in unconscious patients at acute stage of head injury. For such patients, fluid transfusion (polyvinylpyrrolidone, etc.) of $100-300$ c.c. in volume were useful and important procedure which improved the retinal circulation and then clinical improvements were obtained.

2) Clinical improvement ran parallel with circulatory betterment in retina.

3) In case of acute intracranial hematoma, dimness around papilla was observed together with circulatory disturbance in retina, such a change of which advanced to choked disk.

4) There were observed significant changes in retina of post-traumatic syndrome cases.

\section{Surgical Treatment of the Optic Nerve Injury}

Y. Inaba, K. Ishizuka, N. Hoshiko, M. Yanagisawa, T. Nonaka,

M. Fuse, Y. Uchida, T. MiYanaga, A. Yamagami,

K. Ohara and H. Maruta

Dept of Surgery, Tokyo Medicodental College 